\title{
Whole genome comparative analysis of transposable elements provides new insight into mechanisms of their inactivation in fungal genomes
}

\author{
Joëlle Amselem ${ }^{1,2^{*}}$, Marc-Henri Lebrun² and Hadi Quesneville ${ }^{1}$
}

\begin{abstract}
Background: Transposable Elements (TEs) are key components that shape the organization and evolution of genomes. Fungi have developed defense mechanisms against TE invasion such as RIP (Repeat-Induced Point mutation), MIP (Methylation Induced Premeiotically) and Quelling (RNA interference). RIP inactivates repeated sequences by promoting Cytosine to Thymine mutations, whereas MIP only methylates TEs at $C$ residues. Both mechanisms require specific cytosine DNA Methyltransferases (RID1/Masc1) of the Dnmt1 superfamily.

Results: We annotated TE sequences from 10 fungal genomes with different TE content (1-70\%). We then used these TE sequences to carry out a genome-wide analysis of $C$ to $T$ mutations biases. Genomes from either Ascomycota or Basidiomycota that were massively invaded by TEs (Blumeria, Melampsora, Puccinia) were characterized by a low frequency of C to T mutation bias (10-20\%), whereas other genomes displayed intermediate to high frequencies (25-75\%). We identified several dinucleotide signatures at these C to T mutation sites (CpA, CpT, and CpG). Phylogenomic analysis of fungal Dnmt1 MTases revealed a previously unreported association between these dinucleotide signatures and the presence/absence of sub-classes of Dnmt1.

Conclusions: We identified fungal genomes containing large numbers of TEs with many C to T mutations associated with species-specific dinucleotide signatures. This bias suggests that a basic defense mechanism against TE invasion similar to RIP is widespread in fungi, although the efficiency and specificity of this mechanism differs between species. Our analysis revealed that dinucleotide signatures are associated with the presence/absence of specific Dnmt1 subfamilies. In particular, an RID1-dependent RIP mechanism was found only in Ascomycota.
\end{abstract}

Keywords: Transposable elements, Fungi, Repeat induced point mutation, C5-methyltransferase

\section{Background}

Transposable elements (TEs) are mobile genetic element able to transpose and multiply in genomes. A unified TE classification with further subdivisions into subclasses, orders and families has been proposed [1]. The two major subdivisions are class I (retrotransposons) and class II (DNA transposons), which differ according to their mechanism of transposition. Massive TE expansions play a significant role in genome structure, dynamics and evolution

\footnotetext{
* Correspondence: joelle.amselem@versailles.inra.fr

'INRA, UR1164 URGI Research Unit in Genomics-Info, F-78026 Versailles, France

${ }^{2}$ INRA, UR1290 BIOGER, Biologie et gestion des risques en agriculture, Campus AgroParisTech, F-78850 Thiverval-Grignon, France
}

[2]. They shape the genomic landscape by providing novel DNA sequences at various locations, by contributing to chromosomal rearrangements, gene duplications, gene loss and inactivation and by accelerating evolution. Thus, TEs play an important role in adaptation and speciation [3]. In fungi, they have been shown to accelerate the evolution of genes that affect pathogenicity and host range $[4,5]$. The ability of TEs to invade genomes is frequently counterbalanced by defense mechanisms that restrain their expression and mobility [6]. Three defense mechanisms against TEs are known in fungi [7]. Repeat-Induced Point Mutation (RIP) occurs at a premeiotic stage during sexual reproduction. This defense mechanism detects 
DNA sequence duplications and induces irreversible C:G to $\mathrm{T}$ :A mutations at a high rate in these sequences. This fungal-specific defense mechanism was first discovered in $N$. crassa $[8,9]$ and then in a few other fungal species [10]. RIP occurs preferentially at CpA dinucleotide sites in most fungi [9], although other dinucleotide sites including CpG may also be involved [11-13]. RIP requires the RID1 gene, predicted to encode a C5-DNA-Methyltransferase (MTase) of the Dnmt1 family [14]. Dnmt1 enzymes methylate cysteine residues at specific dinucleotide sites (mainly $\mathrm{CpG}$ sites) [15]. The high number of $\mathrm{C}$ to $\mathrm{T}$ mutations associated with RIP is thought to occur by RID1-mediated deamination of methylated cytosine, leading to its replacement with thymine $[10,16]$. Orthologs of this gene have been identified in other Neurospora species [16] including Aspergillus fumigatus and Aspergillus nidulans [17], Fusarium graminearum [18], and Leptosphaeria maculans [19]. The second defense mechanism, called MIP (Methylation Induced Premeiotically), also occurs during sexual reproduction. It was first discovered in Ascobolus immersus and is required for the de novo methylation of cytosine in repeated sequences during meiosis. MIP requires $M A S C 1$, a gene encoding a putative a Dnmt1 cytosine methyl transferase related to RID1 [20-22]. A third defense mechanism, called Quelling, was first identified in $N$. crassa [23]. It involves the RNA interference machinery (AGO, RDE) that suppresses TE expression [24,25].

Another gene-silencing process, the meiotic silencing by unpaired DNA (MSUD) occurring in meiotic cells has been reported. In N. crassa, unpaired DNA causes silencing of all their homologs DNA (paired or not). This process is mediated by putative RNA-directed RNA polymerase (RDRP) [26].

Both RIP and MIP require a Dnmt1 cytosine methyl transferase (MTase) from the same subfamily [14]; therefore, these processes probably rely on similar molecular mechanisms, such as transient de novo methylation of cytosine residues in repeated sequences during sexual reproduction at specific dinucleotide sites. Other known fungal Dnmt1 MTases are involved in cytosine methylation associated with heterochromatin maintenance such as Dim-2 in $N$. crassa [27] and Masc2 in A. immersus [28], both of which belong to a different Dnmt1 subfamily than RID1/Masc1 [22,28]. Dnmt1 MTases normally methylate at CpG dinucleotides [28]. However, Dnmt1 MTases involved in RIP bias may have evolved to recognize dinucleotides other than CpG. In the basidiomycete Microbotryum violaceum, $\mathrm{C}$ to $\mathrm{T}$ mutation bias in TEs occurs preferentially at a trinucleotide TpCpG site [29], suggesting the involvement of a Dnmt1 MTase specific to the CpG di-nucleotide. This mutation bias at CpG di-nucleotide is consistent with the model of mutation via methylation-mediated deamination of 5-methyl cytosine $(5 \mathrm{mC})$ previously describe in human genetic disease [30].
In Ascomycetes, RIP occurs preferentially at dinucleotide CpA sites [13]; however, in A. niger and A. fumigatus RIP may also occur at $\mathrm{CpG}$ sites in TEs in addition to $\mathrm{CpA}$ [11-13], suggesting the involvement of Dnmt1 Mtases recognizing mainly $\mathrm{CpA}$ but also $\mathrm{CpG}$ di-nucleotides. However it has also been suggested that the RIP-like process based on $5 \mathrm{mC}$ methylation followed by deamination may operate in a different manner in basidiomycetes than ascomycetes and that the rid homologue may have diverged during the evolutionary time separating these two phyla [31].

In this study, we conducted a bioinformatics analysis of $\mathrm{C}$ to $\mathrm{T}$ mutation bias in 10 fungal genomes with different TE content, which were recently analysed using the TEdenovo [32,33] and the TEannot [34] from the REPET TE annotation package in the context of international fungal genome projects. We used the consensus sequences of TE families and corresponding TE genomic sequences for each genome obtained from the TEdenovo and the TEannot pipelines, respectively. We performed (i) a genomewide comparison of TE content; (ii) a RIPCAL-based [35] exhaustive search of $\mathrm{C}$ to $\mathrm{T}$ mutations in TEs with their associated dinucleotide sites; and (iii) a functional annotation and phylogenetic analysis of genes encoding RID/ Masc1 and Dim-2/Masc2 Dnmt1 MTases in these 10 genomes. The observed dinucleotide patterns at $\mathrm{C}$ to $\mathrm{T}$ mutation sites in fungal TEs suggest that TE defense mechanisms have different sequence specificities. Our findings also reveal an association between these patterns and the presence of specific Dnmt1 genes. We propose a scenario for the evolution of Dnmt1 subfamilies, in which the RID1-dependent RIP mechanism emerged in Ascomycota after its radiation from Basidiomycota.

\section{Results}

\section{Recent TE invasions in fungi explain the lack of} correlation between genome size and taxonomy

We used the REPET TE annotation package to produce a standardized genome-wide annotation of TEs from 10 fungi (Table 1, Additional file 1) with different lifestyles, including seven ascomycetes (Botrytis cinerea (T4 and B05.10 isolates), Sclerotinia sclerotiorum [36], Blumeria graminis fp. hordeï [37], Leptosphaeria maculans [19], Magnaporthe oryzae [38], Tuber melanosporum [39]) and three basidiomycetes (Puccinia graminis and, Melampsora larici-populina [40] and Microbotryum violaceum (in prep; Microbotryum violaceum Sequencing Project, http:// www.broadinstitute.org). Among the 10 species studied, BcinT4 (genome abbreviation see Table 1) had the lowest TE content (1\% of its genome), whereas Bgra had the highest ( $75 \%$ of its genome Table 1, Figure 1A). Sscl, Mory and Mvio had intermediate TE content (9 to 15\%), close to the average TE content in fungi $(25 \%,[40,41])$. Lmac, Tmel, Pgra, Mlar had high TE content (35-50\%). The 
Table 1 Taxonomy of fungal genomes, source and lifestyle

\begin{tabular}{|c|c|c|c|c|c|c|c|c|}
\hline Fungi kingdom & Strain & Abrev. & Phylum & Class Family & $\begin{array}{l}\mathrm{NCBI} \\
\text { BioProject }\end{array}$ & $\% \mathrm{TE}$ & $\begin{array}{l}\text { Assembly } \\
\text { size }\end{array}$ & Lifestyle \\
\hline Botrytis cinerea T4 & T4 & BcinT4 & Ascomycota & $\begin{array}{l}\text { Leotiomycetes } \\
\text { Sclerotiniaceae }\end{array}$ & 64593 & 0.7 & $39.5 \mathrm{Mb}$ & $\begin{array}{l}\text { Necrotrophic } \\
\text { pathogen }\end{array}$ \\
\hline Botrytis cinerea 05.10 & 05.10 & Bcin0510 & & $\begin{array}{l}\text { Leotiomycetes } \\
\text { Sclerotiniaceae }\end{array}$ & 20061 & 2.2 & $38.8 \mathrm{Mb}$ & $\begin{array}{l}\text { Necrotrophic } \\
\text { pathogen }\end{array}$ \\
\hline sclerotinia sclerotiorum & 1980 UF-70 & Sscl & & $\begin{array}{l}\text { Leotiomycetes } \\
\text { Sclerotiniaceae }\end{array}$ & 20263 & 9.5 & $38.2 \mathrm{Mb}$ & $\begin{array}{l}\text { Necrotrophic } \\
\text { pathogen }\end{array}$ \\
\hline $\begin{array}{l}\text { Blumeria graminis } \\
\text { f. sp. hordeï }\end{array}$ & $\mathrm{DH} 14$ & Bgra & & $\begin{array}{l}\text { Leotiomycetes } \\
\text { Erysiphaceae }\end{array}$ & 28821 & 76.4 & $120 \mathrm{Mb}$ & $\begin{array}{l}\text { Obligate biotrophic } \\
\text { pathogen }\end{array}$ \\
\hline Magnaporthe oryzae & 7015 & Mory & & $\begin{array}{l}\text { Sordariomycetes } \\
\text { Magnaporthaceae }\end{array}$ & 1433 & 11.1 & $40.9 \mathrm{Mb}$ & $\begin{array}{l}\text { Hemibiotrophic } \\
\text { pathogen }\end{array}$ \\
\hline Leptosphaeria maculans & $\mathrm{JN} 3$ & Lmac & & Dothideomycetes & 63129 & 33.3 & $44.9 \mathrm{Mb}$ & $\begin{array}{l}\text { Hemibiotrophic } \\
\text { pathogen }\end{array}$ \\
\hline Tuber melanosporum & Mel28 & Tmel & & $\begin{array}{l}\text { Pezizomycetes } \\
\text { Leptosphaeriaceae }\end{array}$ & 49017 & 60.1 & $123.6 \mathrm{Mb}$ & Symbiotic \\
\hline $\begin{array}{l}\text { Melampsora } \\
\text { larici-populina }\end{array}$ & 98AG31 & Mlar & Basidiomycota & $\begin{array}{l}\text { Pucciniomycetes } \\
\text { Melampsoraceae }\end{array}$ & 46711 & 51.7 & 101.1 Mb & $\begin{array}{l}\text { Obligate biotrophic } \\
\text { pathogen }\end{array}$ \\
\hline $\begin{array}{l}\text { Puccinia graminis } \\
\text { f. sp. tritici }\end{array}$ & CRL 75-36-700-3 & Pgra & & $\begin{array}{l}\text { Pucciniomycetes } \\
\text { Pucciniaceae }\end{array}$ & 66375 & 46.4 & $81.6 \mathrm{Mb}$ & $\begin{array}{l}\text { Obligate biotrophic } \\
\text { pathogen }\end{array}$ \\
\hline Microbotryum violaceum & P1A1 Lamole & Mvio & & $\begin{array}{l}\text { Pucciniomycetes } \\
\text { Mycrobotryaceae }\end{array}$ & 41281 & 14.1 & $25.2 \mathrm{Mb}$ & $\begin{array}{l}\text { Obligate biotrophic } \\
\text { pathogen }\end{array}$ \\
\hline
\end{tabular}

genomes of fungi with high TE content (>35\%) were larger than those of typical fungi or related species with low TE content (Figure 1A). The large TE content of Bgra (75\%) substantially affects the size and organization of its genome: the Bgra genome is $120 \mathrm{Mb}$, which is $80 \mathrm{Mb}$ larger than that of the related species Bcin and Sscl, contains only $30 \mathrm{Mb}$ of coding space, compared to $40 \mathrm{Mb}$ in other Ascomycetes species, and many of its genes are surrounded by TE rich regions. The highly related species Blumeria graminis formae speciales tritici also has a genome with high TE content (90\%) as do other related species from the Erysiphales (80-90\%) [42]. The occurrence of TE functional categories greatly differs between species (Figure 1B) and is independent of their taxonomic relationship (Table 1), except for the related rust fungi Pgra and Mlar [40]. For example, Tmel, Mory and Lmac are characterized by a high proportion of LTR retrotransposons (60-80\% of TE content), although they are classified in very distantly related taxonomic orders, whereas the main TE categories in more related species such as Mory and Sscl are very different: LTRs make up 10\% and $60 \%$ of TEs in Sscl and Mory, respectively and TIR TEs make up $30 \%$ and $20 \%$ of TEs in Sscl and Mory, respectively (Figure 1B). We noted similar differences between highly related species such as Bcin and Sscl. LINE and Helitron TEs were detected in Sscl (15\% and 5\% of TE content, respectively), but not in Bcin, whereas the proportion of LTR TEs was lower in Sscl (10\%) than in Bcin (36\%). The differences in TE content and TE categories among related species, such as BcinT4 and Bcin0510,

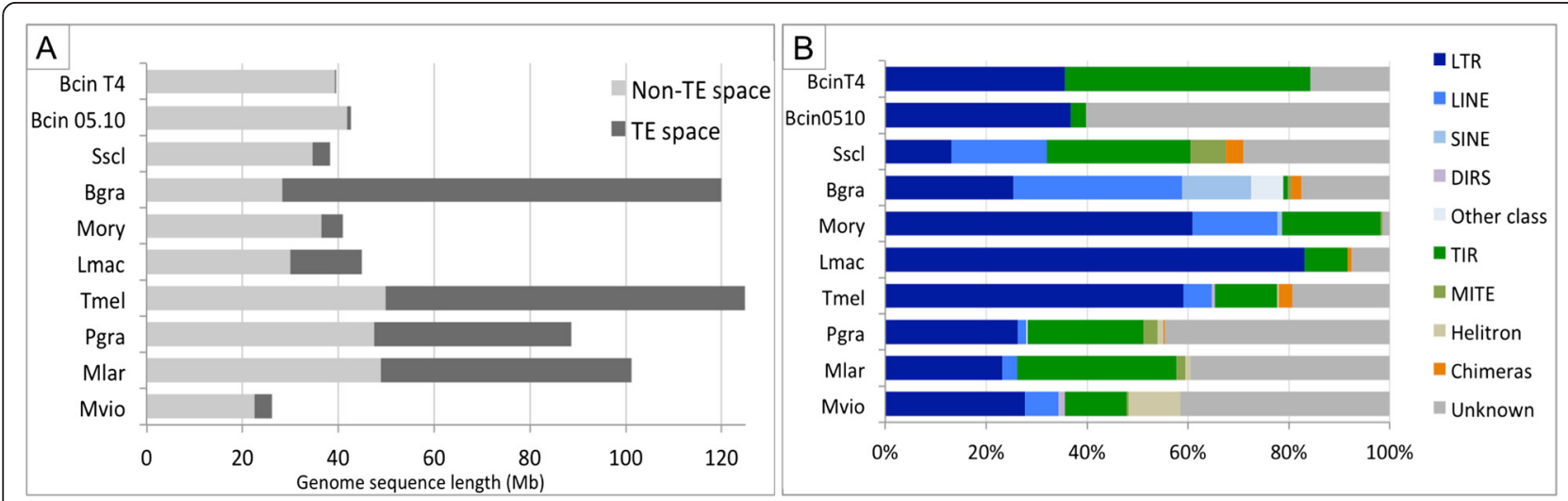

Figure 1 Distribution of TEs in fungal genomes. (A) TE vs non-TE space, (B) Percentage of different categories of TEs: class I retrotransposons (LTR, LINE, SINE, DIRS) and class II DNA transposons (TIR, MITE, Helitrons), unclassified (Unknown) and chimeras of TE consensus sequences. 
and Sscl and Bgra, are presumably due to relatively recent TE invasions, as suggested previously $[36,37,39]$. For example, differences in the composition of TEs between Sscl and Bcin are due to the recent invasion of a few families of class II TIR DNA and LINE TEs in Sscl. These subfamilies of Sscl TIR TEs are composed of almost identical copies dispersed in the genome, whereas other subfamilies of Sscl TIR TEs are highly polymorphic [36].

\section{Analysis of TE G:C content reveals multi-modal distributions} One major consequence of RIP involves genome G:C content, because RIP increases the A:T content of mutated TE copies. As a result, when TEs are clustered in large blocks, the RIP-mediated mutation of C:G to A:T generates A:T rich isochores, as observed in Lmac [19]. We sought to identify A:T rich regions associated with TEs; therefore, we compared the G:C content of high quality TEs (see Methods, hereafter referred to as TEcpHQ) or low quality TEs (hereafter referred to as TEcpLQ) with the $\mathrm{G}: \mathrm{C}$ content of the whole genome across $2 \mathrm{~Kb}$ sliding windows denoted as GSW. Lmac was the only species of our sample that displayed a bimodal GSW. The peak at 36\% G:C corresponds to A:T-rich isochores composed mainly of RIPed TE copies (Figure 2), which have been described previously [19]. Other genomes displayed a unimodal GSW with a peak around $45-50 \%$ G:C, with the exception of Mvio (60\%) and the negative control Atha (42\%). The distribution of G:C content in TEs frequently differed from that in the GSW. In the negative control Atha, the G:C content of TEcpHQ was bimodal. The low TEcpHQ values (20\%) probably correspond to heavily mutated ancestral TEs that tend to be depleted in C:G sites because they are highly methylated [43,44]. In genomes invaded by TEs (Mvio, Tmel, Mlar, Pgra, Bgra) the distribution of G:C content was similar between TEs (TEcpHQ) and the whole genome (GSW, Figure 2). However, the high TE content of these genomes clearly introduces a bias because TE content makes up a larger proportion of genomic space than non-TE content. In other genomes, the distribution of $\mathrm{G}: \mathrm{C}$ content between TEs and the whole genome was very different, in particular for TEcpHQ. For example, TEcpHQ elements in BcinT4 had four peaks of GC content; one of these peaks $(44 \% \mathrm{G}: \mathrm{C})$ was very similar to the whole genome (GSW) peak, whereas the three other peaks comprised two groups of TEs with low G:C content (20 and 40\%) and one group with high G:C content (55\%). Mory showed a similar profile consisting of two peaks (40 and $60 \%$ ) surrounding another peak at 50\% corresponding to the whole genome GC content. TEs peaks with low $\mathrm{G}: \mathrm{C}$ content in BcinT4 may correspond to TEs copies that have undergone RIP-associated $\mathrm{C}$ to $\mathrm{T}$ mutations at various rates. Indeed, the TEcpHQ peak at $20 \% \mathrm{G}$ :C content in BcinT4 comprises AT-rich TE copies that are even more abundant among TEcpLQ elements (Figure 2). These highly degenerated TEs may result from multiple rounds of RIP leading to the mutation of all their target C:G sites.

\section{Dinucleotide signatures associated with $\mathrm{C}$ to $\mathrm{T}$ mutation sites in TEs differ among fungi and TE families}

The mutation resulting from RIP is a transition (C:G to $\mathrm{T}: \mathrm{A})$. We used multiple sequence alignment (MSA) to compare genomic TE copies with the consensus TE sequence of each family to search for mutational biases and identify RIP-associated signatures in fungal TEs. If all possible mutations were equally likely, we would expect twice as many transversions (Tv) as transitions (Ti), given a random mutation $\mathrm{Ti} / \mathrm{Tv}$ ratio of 0.5 . We used MSAs from Atha TEs [32,45] as negative controls of RIP-associated biases because RIP has not been observed in plants. We found that the mean $\mathrm{Ti} / \mathrm{Tv}$ ratio of Atha TE families was equal to 1.2 (all TEs, Figure 3). Genome-wide analysis of Bgra, Pgra and Mlar TEs showed that their average $\mathrm{Ti} / \mathrm{Tv}$ ratio was similar to that of Atha (all TEs = 1.2 to 1.7). TEs copies from all other fungal genomes displayed a higher mean $\mathrm{Ti} / \mathrm{Tv}$ ratio (all $\mathrm{TEs}=2$ to 25.7). We selected TE genomic copies with a Ti/Tv $>2$ (Figure 3), which is a stringent threshold compared to the Atha negative control $(\mathrm{Ti} / \mathrm{Tv}=1.2)$. Using these $\mathrm{TE}$ sequences, we searched for dinucleotides associated with $\mathrm{C}$ to $\mathrm{T}$ mutation sites. Lmac TEs were used as a positive control for a RIP-associated signature [19]. We first observed that the percentage of $\mathrm{TE}$ copies with a high $\mathrm{Ti} / \mathrm{TV}$ ratio varied widely from genome to genome (Figure 3). Most Lmac TEs $(96 \%)$ had a high $\mathrm{Ti} / \mathrm{Tv}$ ratio $(\mathrm{Ti} / \mathrm{Tv}>2$, Figure 3$)$, as reported previously [19]. By contrast, the percentage of TEs with a Ti/Tv ratio $>2$, was low (14 to $22 \%$ ) and similar to that of the negative control Atha $(11 \%)$ in the three species (Bgra, Mlar, Pgra) massively invaded by TEs, suggesting that they are deficient for RIP. The remaining species had an intermediate proportion of TEs (40 to 67\%) with a $\mathrm{Ti} / \mathrm{Tv}$ ratio $>2$ (Figure 3). We next searched for dinucleotide signatures at $\mathrm{C}$ to $\mathrm{T}$ mutation sites in TEs with a Ti/ Tv ratio $>2$ (Figure 4). As expected, we found that the vast majority (90\%) of Lmac TE C to T mutation sites was associated with a CpA dinucleotide (Figure 4). We also found this canonical RIP-associated dinucleotide signature in a large fraction $(40 \%)$ of Sscl TEs. However, we identified other dinucleotide signatures $(\mathrm{CpT}, \mathrm{CpG})$ at $\mathrm{C}$ to $\mathrm{T}$ mutation sites in TEs from other fungal genomes. In some fungal species, these were the only signatures present in TEs, whereas in other species they were found in addition to CpA signatures. In BcinT4, BcinB0510, Sscl, and Mory, we identified a large number of TEs with a $\mathrm{CpT}$ signature (10-40\% of TEs) in addition to CpA signatures $(10-35 \%$ of TEs). In the TE-rich Tmel genome, many TE copies (67\%) displayed a $\mathrm{Ti} / \mathrm{Tv}$ ratio $>2$. The $\mathrm{C}$ to $\mathrm{T}$ mutation sites of 


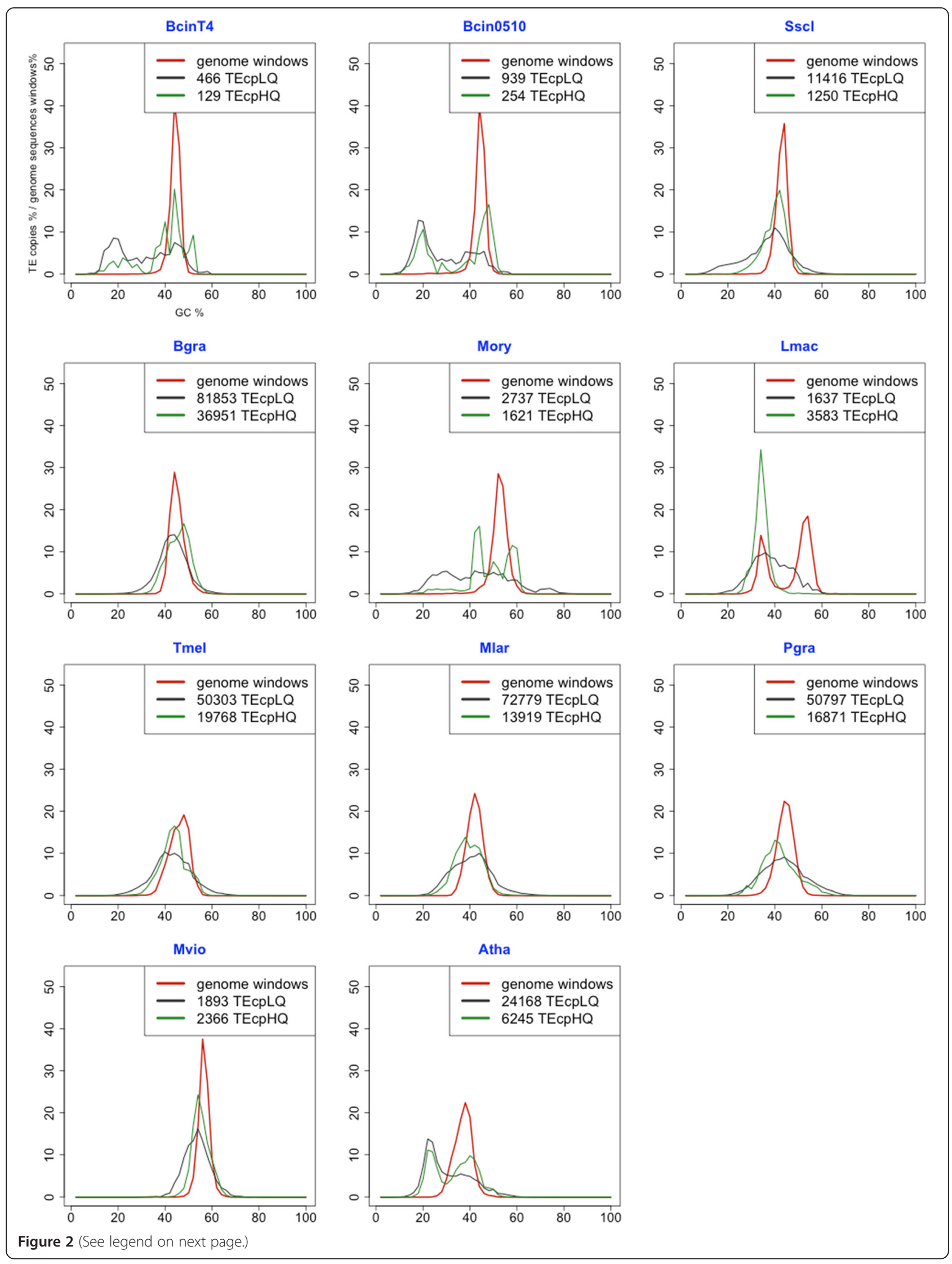


(See figure on previous page.)

Figure 2 Comparison of GC content (\%) in TE copies and genome sliding windows. GC content in TE copies: GC content in Low quality

(TEcpLQ, gray) and High quality (TEcpHQ, green) TEs and in genome sliding windows (red) was calculated with sliding windows (windows = 2 Kb, increment $=2 \mathrm{~Kb}$ ). Y axis: TE copies\% or Sliding windows\%. X axis: GC\%.

Tmel TEs displayed predominantly a CpG signature (40\% of TEs) in addition to a CpA signature (25\% of TEs). In Mvio, a large number of TE copies (40\%) displayed a Ti/ Tv ratio $>2$. The $\mathrm{C}$ to $\mathrm{T}$ mutation sites of Mvio TEs displayed only a CpG signature (37\% of TEs, Figure 3). In genomes with high TE content (Bgra, Pgra and Mlar), the number of TE copies that displayed only a CpG signature at their $\mathrm{C}$ to $\mathrm{T}$ mutation site was small (Figure 4).

\section{Dinucleotide signatures at $\mathrm{C}$ to $\mathrm{T}$ mutation sites in fungal TEs are associated with specific Dnmt1 subfamilies}

We next addressed whether the differences in $\mathrm{C}$ to $\mathrm{T}$ mutation bias in TEs and their associated dinucleotide signatures among fungi could be explained by the presence/absence in these various species of different genes involved in this process. RIP and MIP require cytosine methyltransferases of the Dnmt1 family; therefore, we searched for 25 fungal genomes (included the 10 fungal genomes studied) for genes encoding Dnmt1 MTases. Forty-four proteins containing the cytosine specific methyltransferase Panther domain (PTHR10629) were recovered from these 25 genomes. This domain overlaps on both ends the C5-cytosine methyltransferase PFAM domain (PF00045), which is specific to the cytosine DNA MTase family (Figure 5B). Phylogenetic analysis of the PTHR10629 domain extracted from the 44 Dnmt1 protein sequences (Figure 5A) showed that these proteins clustered into two very distinct clades. The first clade (class I), contains proteins related to RID and Masc1 whereas the second clade (class II) contains proteins related to Dim2 and Masc2. This second clade is composed of two subclasses (IIA and IIB), corresponding to proteins related to either Masc2 or to Dim2, respectively. Interestingly, all the genomes with $\mathrm{CpA}$ or $\mathrm{CpT}$ dinucleotide signatures in TEs, had at least one gene coding for a protein of the Masc1/RID family (Dnmt1 class I, Figure 5A, Table 2). All these genomes belong to species of Ascomycota (Additional file 1). The three genomes that displayed only TEs with a CpG dinucleotide signature (Mvio, Mlar and Pgra) contained only one gene coding for a Dnmt1, a class IIA protein related to Masc2 (Figure 5A, Table 2). These three organisms belong to the Basidiomycota.

Protein domain annotation of the 44 Dnmt1 proteins revealed that the proteins belonging to the classes and subclasses established above (phylogeny based on the PTHR10629 domain) showed extensive synapomorphy in their domain composition (Figure 5B). Phylogenetic analysis and taxonomic distribution suggest that the C5_MTASE_2 C-terminal amino-acid signature (PS00095) was most likely present in the common ancestor of both classes (most parsimonious hypothesis). By contrast, the Bromo-Adjacent Homology (BAH) domain, which is commonly found in chromatin-associated proteins and usually present in a duplicated form [46], and the N-terminal Dnmt1-RFD (PF12047), which is a methyltransferase replication foci domain of the Dnmt1 protein required for methylation of the correct residue (CpG methylation site) [47], were detected only in class 2 Dnmt 1 proteins (Dim2/Masc2). Several domains were irregularly distributed among fungal Dnmt1 proteins. For instance, the PROSITE domain C5_MTASE_1 (PS00094), which is part of the PF00145 domain (i.e. the PFAM equivalent of PTHR10629), was detected in almost all (11 out of 12) Dnmt1 class IIB (Dim-2) proteins, but only in a subcluster (comprising 4 out of 12) of class IIA proteins that are highly related to Masc2. This domain is absent in almost all (18 out of 19) Dnmt1 class I (Masc1/RID proteins). By contrast, C5_MTASE_2 (PS00095), a C-5 cytosine-specific DNA methylase C-terminal aminoacid signature, was found in $50 \%$ of the Masc1/RID proteins and in class II proteins, this domain was restricted to the Masc2-like class IIA subcluster (Figure 5). Thorough examination of the organization of the predicted DNA MTase domain (PF00145) in fungal Dnmt1 homologs revealed that it is on average smaller (240 aa) in class I proteins than in class II proteins (400 aa). This difference is due to a large region upstream from the C-terminal catalytic domain that is present only in the Dnmt1 class II proteins. Another amino-acid probably lost the gene, the C5METTRFRASE (PR00105) from the PRINTS fingerprint protein database, was present in almost all (80\%) Dnmt1 proteins. Nevertheless, it is probable that these three patterns (C5_MTASE_1, C5_MTASE_2 and C5METTRFRASE) were probably present in a common ancestor of the two classes because we found relics of these motifs in all 44 proteins analyzed (Additional file 2). We also used the MEME tool to identify conserved motifs in fungal Dnmt1 proteins. This analysis revealed that 19 out of 30 conserved motifs where specific to either Dnmt1 class I or class II proteins: five motifs $(15,17,20,26,29)$ were present only in Dnmt1 class I proteins whereas the other 14 motifs were present only in Dnmt1 class II proteins (Additional file 3). The five motifs specific to Dnmt1 class I proteins are not part of any known functional domain. Among these five motifs specific to RID/Masc1-like proteins, one (motif 29) was only found in Bcin, Sscl and Mory (Additional file 3). This domain may be involved in the $\mathrm{CpT}$ di-nucleotide signature specific to 

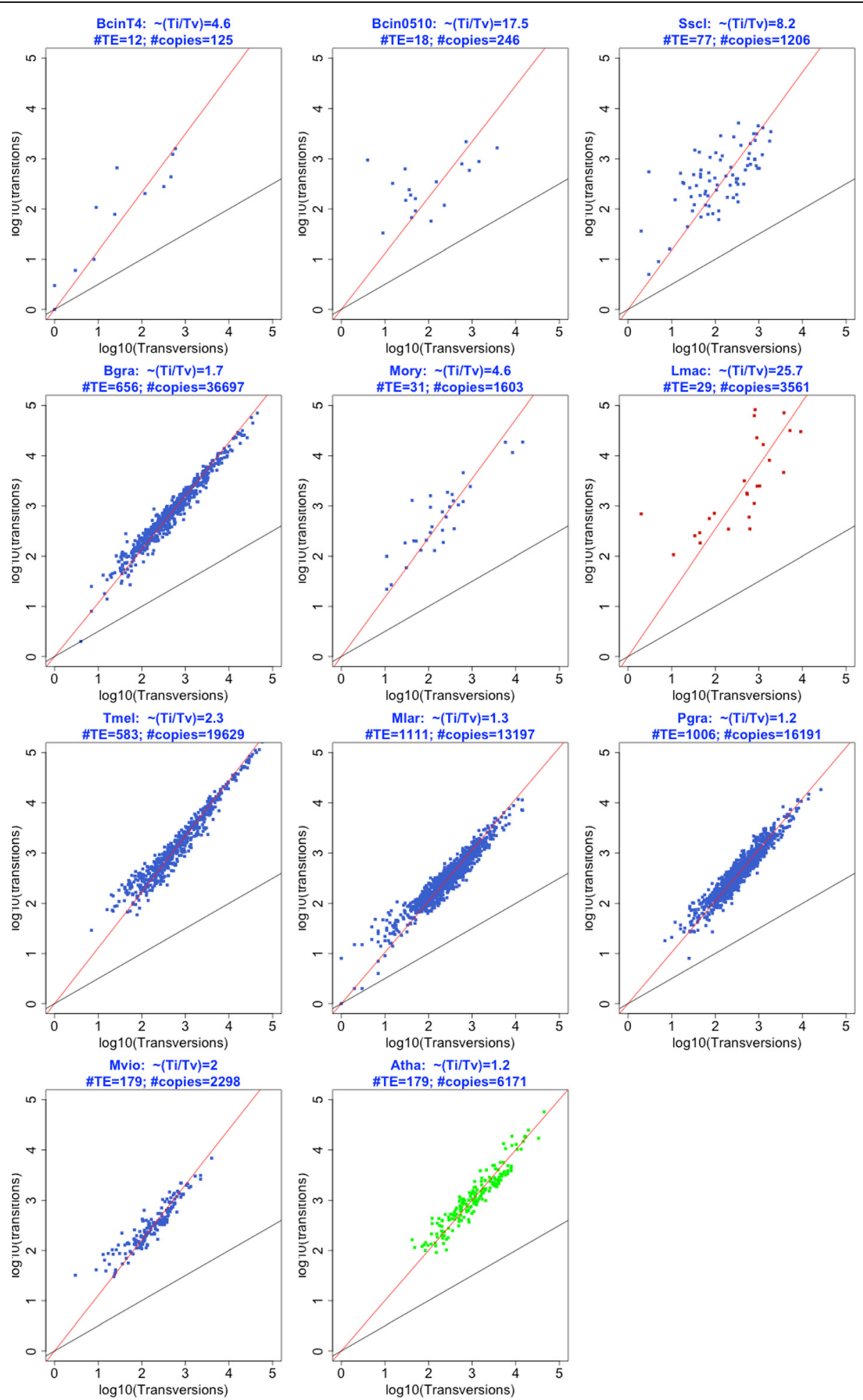

Figure 3 Transition and transversion mutation rates in TE copies from fungal genomes. Transition (Ti, Y axis, log 10) and Transversion (TV, X axis, log 10). Mutation rates were calculated using RIPCAL by comparing each TE copy with the TE consensus sequence or the genomic copy with the highest GC content through multiple alignments. Each dot corresponds to the log 10(sum Ti) vs. $\log 10($ sum TV) of a TE family. (Ti/TV) is the mean of Ti/Tv for all the families in the analysis. The black line corresponds to the linear regression line between the two series (Ti vs TV). The red line corresponds to an equiprobable mutation rate $(\mathrm{Ti} / \mathrm{TV}=0.5)$. The $\mathrm{Lmac}$ and Atha dot plots are red and green respectively to highlight the pattern of positive and negative controls respectively. 


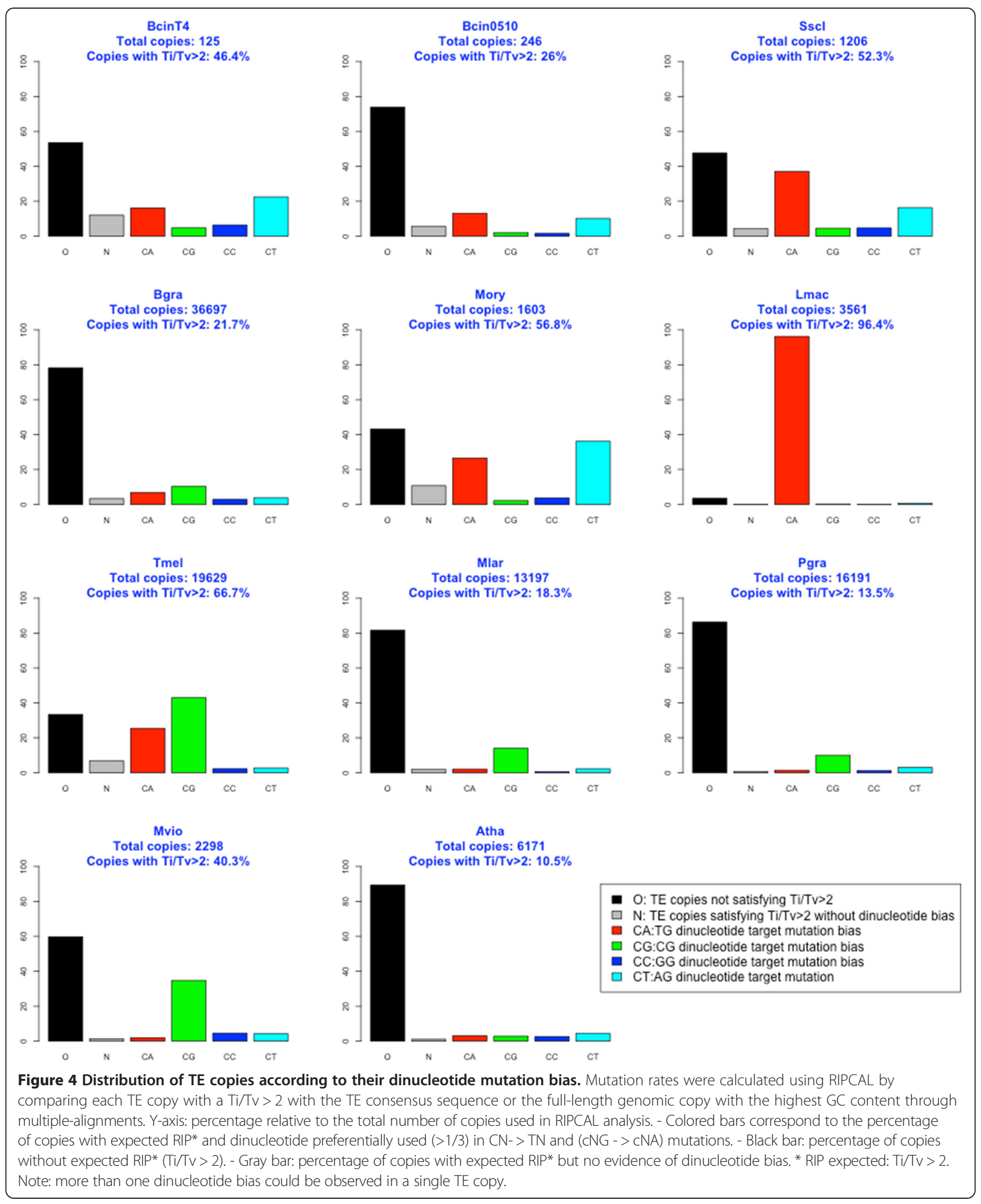

these three genomes. Among the 14 motifs only present in Dnmt1 class II proteins, three (motifs 16, 27 and 28) are specific to class IIA (Masc2 proteins). Motif 16 is located in the BAH domain whereas motifs 12 and 28 are located in the C5-MTase domain. Seven other motifs are specific to the class IIB (including Dim-2) proteins 


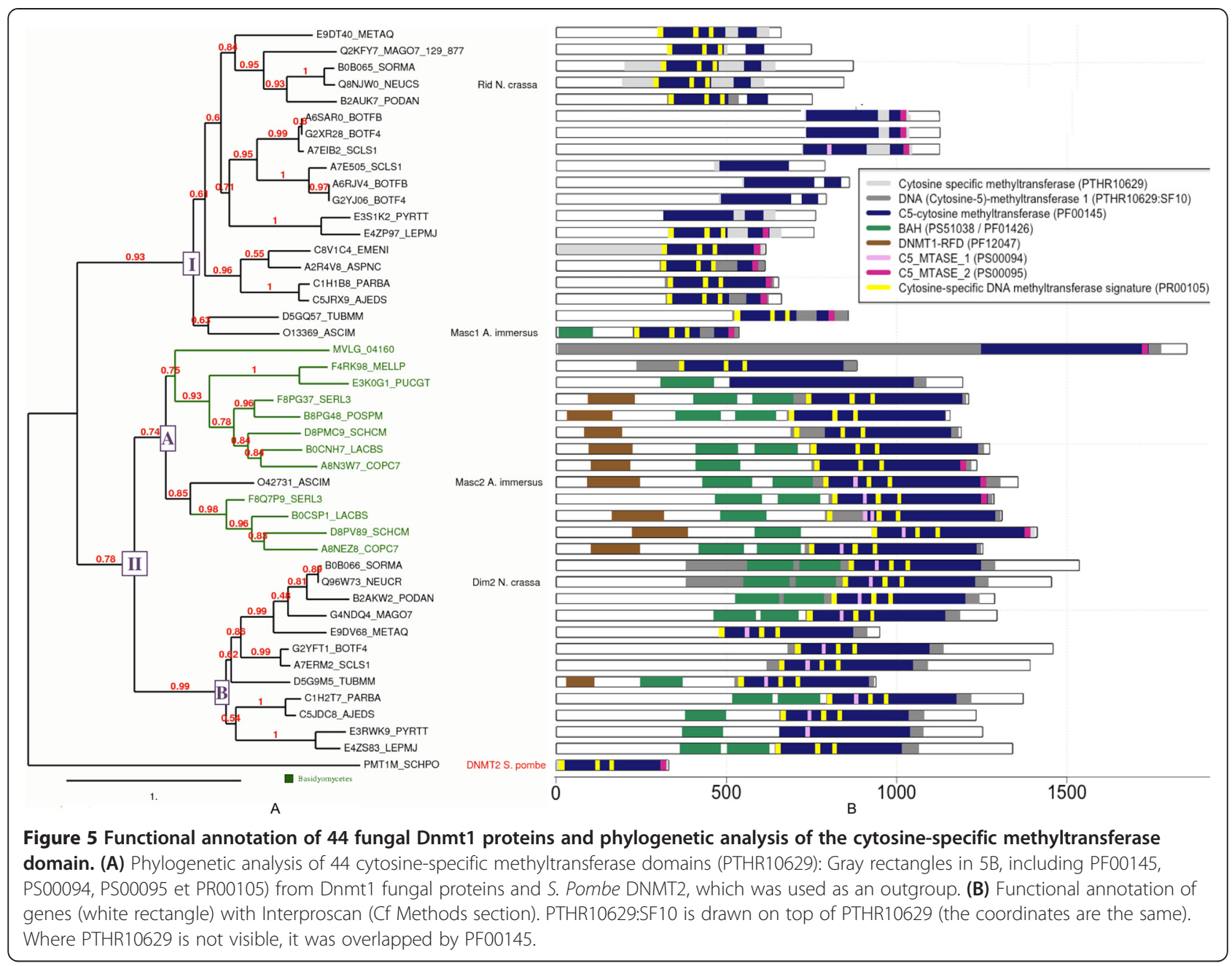

(motifs 25, 22, 18, 28, 21, 30 and 19; from 5' to 3' respectively). Motif 21 is located in the BAH domain and motif 19 is located in the C5_MTase domain. Motifs 25, 22 and 18, which are upstream from the C5-MTase domains, are located around the motif 11 that is specific to the Dnmt1-RFD domain. This domain is present in most of class IIA proteins but only in one class IIB protein (D5G9M5_TUBMM) (Additional file 4). These results show that the evolution of sequences located in or around the DNMT1-RFD and BAH domains are compatible with the phylogenetic analysis of the C5-MTase domain.

\section{Discussion}

Recent TE invasions of fungal genomes explain the lack of correlation between genome size and taxonomic relationships

We were able to annotate successfully TE copies, even when they were small and degenerated. The TE consensus sequence provided by the TEdenovo detection and classification pipeline was very similar to the ancestral sequence of the TE family. The annotations obtained with these consensus libraries provided a quasi-exhaustive set of copies that could be used to search for methylation signatures of repeat-based silencing processes. Except for Pgra and Mlar, which are characterized by similar categories of TEs and an almost equivalent TE content and distribution of class I and class II elements, we did not find any correlation between the distribution of TEs and taxonomic relationships. The main difference in the composition of TEs in the two related fungi Botrytis and Sclerotinia is due to the lack of LINE elements in Bcin (Figure 1B). The difference in their TE content ( 8 fold higher in Sscl, Figure 1) is almost certainly due to a recent invasion of class II TIR elements [36].

The frequency of $\mathrm{C}$ to $\mathrm{T}$ mutation bias in TEs is correlated with genome size and organization

Our RIP signature analysis in Lmac revealed the presence of AT-rich genome blocks composed of highly RIPed copies, thus confirming our previous findings in this species. Similar AT-Rich isochore-like islands were also recently found in repeated regions of the Colletotrichum 
Table 2 Association between dinucleotide signatures preferentially found at C:G to T:A mutation sites and presence/ absence of DNMT1 genes

\begin{tabular}{|c|c|c|c|c|c|}
\hline \multirow[t]{2}{*}{ Organism } & $\begin{array}{l}\text { Predominant } \\
\text { C to } \mathrm{T}\end{array}$ & $\begin{array}{l}\text { Secondary } \\
\text { C to T }\end{array}$ & $\begin{array}{l}\text { Dnmt1 class I } \\
\text { RID/Masc1 }\end{array}$ & $\begin{array}{l}\text { Dnmt1 class IIA } \\
\text { Masc2 }\end{array}$ & $\begin{array}{l}\text { Dnmt1 class IIB } \\
\text { Dim2 }\end{array}$ \\
\hline & $\begin{array}{l}\text { Dinucleotide } \\
\text { mutation bias }\end{array}$ & $\begin{array}{l}\text { Dinucleotide } \\
\text { mutation bias }\end{array}$ & $\begin{array}{l}\text { Uniprot accession no. } \\
\text { of similar protein }\end{array}$ & $\begin{array}{l}\text { Uniprot accession no. } \\
\text { of similar protein }\end{array}$ & $\begin{array}{l}\text { Uniprot accession } \\
\text { no. of similar protein }\end{array}$ \\
\hline Lmac & $\mathrm{CpA}$ & - & E4ZP97_LEPMJ & $\mathrm{Nd}$ & E4ZS83_LEPJM \\
\hline Mory & CpT & CpA & Q2KFY7_MAG07 & $\mathrm{Nd}$ & $\mathrm{Nd}$ \\
\hline \multirow[t]{2}{*}{ BcinT4 } & CpT & CpA & G2XR28_BOTF4 & $\mathrm{Nd}$ & G2YFT1 \\
\hline & & & G2YJ06_BOTF4 & & \\
\hline \multirow[t]{2}{*}{ BcinB05.10 } & $\mathrm{CpA}$ & CpT & A6SARO_BOTBB & $\mathrm{Nd}$ & $\mathrm{Nd}$ \\
\hline & & & A6RJV4_BOTFB & & \\
\hline \multirow[t]{2}{*}{ Sscl } & $\mathrm{CpA}$ & СpT & A7E1B2_SSCL1 & $\mathrm{Nd}$ & A7ERM2_SSCL1 \\
\hline & & & A7E505_SSCL1 & & \\
\hline Bgra & ${ }^{1} \mathrm{CpG}$ & - & $\mathrm{Nd}$ & $\mathrm{Nd}$ & $\mathrm{Nd}$ \\
\hline Tmel & CpG & $\mathrm{CpA}$ & D5GQ57_TUBMM & $\mathrm{Nd}$ & D5G9M5_TUBMM \\
\hline Pgra & ${ }^{1} \mathrm{CpG}$ & - & $\mathrm{Nd}$ & E3KOG1_PUCGT & $\mathrm{Nd}$ \\
\hline Mlar & ${ }^{1} \mathrm{CpG}$ & - & $\mathrm{Nd}$ & F4RK98_MELLP & $\mathrm{Nd}$ \\
\hline Mvio & $\mathrm{CpG}$ & - & $\mathrm{Nd}$ & MVLG_04160 & $\mathrm{Nd}$ \\
\hline
\end{tabular}

Results are shown if the proportion of copies exhibiting a mutation bias was over $10 \%$.

${ }^{1}$ Very weak bias (10\% of copies). Nd: not determined.

graminicola genome [48]. These organisms may be examples of successful TE invasions (35 and 22\% TE content for Lmac and C. graminicola, respectively) counterbalanced by an efficient RIP silencing mechanism. We also noticed that genomes with a lower TE content, such as B. Cinerea T4 and B0510 isolates (0.7 and 2\% $\mathrm{TE}$ content, respectively) have many AT rich relics of TE copies that are probably signatures of ancient RIP (Figure 2, TEcpLQ). We also found an interesting correlation between fungal lifestyles and massive TE expansion; the obligate biotrophs Bgra, Mlar and Pgra and the symbiote (ectomycorrhiza) Tmel had higher TE loads than the remaining studied fungi which are all necrotrophs.

\section{Dinucleotide mutation signatures at $\mathrm{C}$ to $\mathrm{T}$ mutation sites in fungal TEs are associated with specific DNA methyltransferases}

In four fungal species (B. cinerea, S. sclerotiorum, $M$. oryzae and T. melanosporum), TE copies showed mutational biases at two different dinucleotides that could be signatures of two different mechanisms (Table 2). All four species had a $\mathrm{CpA}$ dinucleotide signature, like in $\mathrm{N}$. crassa where the RIP was experimentally observed [8] or in L. maculans where it the high level of RIP was detected in-silico [19]. T. melanosporum had a CpG signature, whereas a CpT signature was found in the three other species. A fifth species, L. maculans exhibited an extensive $\mathrm{CpA}$ signature suggestive of a high rate of RIP, and showed no other dinucleotide signature. A RID/Masc1-like gene was present (in one or two copies) only in the five species exhibiting a CpA signature in their TE copies, (which was accompanied by $\mathrm{CpT}$ bias in some species). We did not find any gene of the Dnmt1 family in B. graminis $f$. $s p$. hordei. The absence of genes responsible for repeat silencing probably explains the large expansion of TEs in this fungus, as well as in the other formae speciale of wheat, B. graminis triticeae [42]. B. graminis lost half of its genes during its evolution towards an obligate biotrophic lifestyle [37]. It probably also lost the gene(s) responsible for RIP. Indeed, we found traces of RIP in a very small number of TE copies that exhibit the CpA dinucleotide target signature. The RIP of these TE copies probably predates the loss of the RIP gene. Three species (M. violaceum, M. larici-populina and P. graminis) showed only a CpG mutation signature in their TE copies. In these genomes, we did not find any gene belonging to the Dnmt1 class I RID/Masc1-like phylogenetic group (Table 2); instead, we found one gene belonging the Dnmt1 class IIA (including Masc2 gene). This suggests that Dnmt1 class IIA proteins (including Masc2) are involved in a mechanism occurring preferentially at CpG target sites. Indeed, the three species $M$. violaceum, M. larici-populina and P. graminis (all basidiomycetes) of our study exhibited a CpG signature (40\% of Mvio TE copies and 10\% of both Pgra and Mlar TE copies) and possessed a Masc2-like gene, but not any other Dnmt1-like gene. To date, no published analysis has associated the dinucleotide targets of transition mutations to potential genes and functional domains involved in these mutations. 


\section{Evolution of Dnmt1 subgroup proteins}

Here we showed that a part of the PFAM Domain PF00145 specific to DNA methyltransferase (C5_MTASE) is missing from all the RID/Masc1-like proteins (Figure 5B; Additional file 2). In addition, we found four motifs specific to these genes (Additional file 3, Motif 15, 17, 20, 26). The loss of part of the C5_MTASE domain associated with the acquisition of these four motifs may have resulted in the specialization of the gene responsible for the RIP process, the efficiency of which depends on the organism (Figure 3). A fifth motif (Additional file 3, Motif 29) only present in B. cinerea, S. sclerotiorum and M. oryzae could be involved in the recognition of the dinucleotide target $\mathrm{CpT}$ because this signature was detected only in TE copies from these species. The specialization of RID and Dim-2 probably occurred after the radiation between the basidiomycota and ascomycota phyla. Indeed, Masc2-like genes (class IIA phylogenic subgroup, Figure 5A) were the only Dnmt1 genes in basidiomycetes and we found neither RID/Masc1 nor Dim-2 genes in this phylum. This suggests that RIP does not target CpG dinucleotides. In Tmel where was found a $\mathrm{CpG}$ signature in addition to $\mathrm{CpT}$ it was recently showed that a high fraction of transposons with methylated cytosine had a strong preference for $\mathrm{CpG}$ sites [49]. In 2011, Clutterbuck [13] proposed that the mechanism responsible for $\mathrm{C}$ to $\mathrm{T}$ mutations at $\mathrm{CpG}$ target sites in fungi was probably cytosine methylation followed by the deamination of the methyl-cytosines. However a correlation between this process and the genes that are potentially involved has not yet been reported. Although we did not find any RID-like gene or Dnmt1 gene in B. graminis, we found evidence of dinucleotide target signatures such as CpA and CpG $(<10 \%$ of TE copies), showing that these genes were probably active before they were lost. Surprisingly, Masc2 is the only gene found in an ascomycete among the class IIA group of Dnmt1 proteins. We thus hypothesize that the specialization of this gene in only one ascomycete (out of the 16) and basidiomycetes (in our phylogenetic analysis) reflects a convergent evolution (i.e. a natural selection that favors similar function in spite of different ancestor).

\section{Conclusions}

In this article, we provide new insights into RIP and other related process involving the action of $\mathrm{C5}$-Cytosine DNA methyl-transferases during repeat silencing mechanisms in fungi. The absence of RIP or its low efficiency in fungal genomes appears to be responsible for the accumulation of TEs, which increase the size of the genome. We also propose a new classification system for fungal Dnmt1 proteins based on rigorous functional annotation of domains and motifs and phylogeny of the cytosine-specific methyltransferase domain. We show that $\mathrm{CpA}$ and $\mathrm{CpT}$ dinucleotides are probably targeted by a RIP process involving the RID gene, whereas CpG dinucleotides are probably targeted by another methylation-based process involving a Masc2-like gene. The signatures of $\mathrm{CpG}$ to TpG mutations found in some TE copies strongly suggest a mechanism of methyl-cytosine deamination following de novo methylation induced by a process similar to MIP. In addition, we found that the RID protein of three species (B. cinerea, S. sclerotiorum and M. oryzae) containing signatures of $\mathrm{CpT}$ bias possessed a specific motif, suggesting that the RID gene in these three species underwent specialization to recognize this target. We propose that the RIP process and the specialization of the RID gene appeared after the radiation of Basidiomycota and Ascomycota phyla.

\section{Methods}

\section{TE consensus and genomic copy resources}

Datasets of TE families were obtained through several genome sequencing projects that we were involved in. The REPET TEdenovo pipeline [32] was used to detect TEs in genomic sequences and to provide a consensus sequence for each family. TEs were then classified according to structural and functional features (LTR, TIR, RT, transposases, polyA tail) and similarities with characterized TEs from the Repbase Update database [50]. The REPET TEannot pipeline [34] and the previously obtained TE consensus libraries were used to annotate TE genomic copies, including nested and degenerated ones, in each of the 10 genomes. Basic TE annotations have already been published for most of these species, which included: (i) six ascomycetes: Botrytis cinerea T4 (BcinT4 and B05.10 isolates (Bc0510) and Sclerotinia sclerotiorum (Sscl) [36], Blumeria graminis sp. hordeï (Bgra) [37], Leptosphaeria maculans (Lmac) [19] and Tuber melanosporum (Tmel) [39]; and (ii) three basidiomycetes: Puccinia graminis sp. Triticeae (Pgra), Melampsora larici-populina (Mlar) [40] and Microbotryum violaceum (Mvio), (in prep.). We also used TEs from Arabidopsis thaliana (TAIR9) that were annotated with REPET [32]. A thaliana was used as negative controls of RIP-associated biases because RIP has not been observed in plants.

\section{TE annotation refining}

Datasets of TE families (consensus + genomic TE copies) used in the reported analyses were refined in a second TEannot pipeline iteration that was carried out with the improved REPET V2 release. We filtered out poorly defined and redundant TE consensus sequences showing no full-length copies (i.e. copies covering more than $95 \%$ of the consensus sequence) in the genome. Hence, we decreased the complexity of our consensus libraries, and increased the number of TE copies found by some consensus sequences, without any significant loss of sensitivity. We also re-launched PASTEC [33] 
the new TE classifier implemented in the REPET V2 TEdenovo pipeline. TE consensus libraries and annotations in genomes are available at: https://urgi.versailles. inra.fr/download/fungi/TEs/.

\section{Search for RIP-like signatures and dinucleotide targets} involved in transition mutations in genomic TE copies

We identified RIP signatures by comparing each TE genomic copy with the consensus used to annotate it. When TEs have highly diverged during evolution through a RIP-mediated irreversible process the consensus could be more "Ripped" than some of TE copies in the genome because mutations occurring in a given sequence are likely to be removed by the "base-pair majority rule" used to build the consensus. In such a case the copy with the Highest GC content is used to calculate the transition mutations. All TE families (TE consensus with their annotated TE copies) satisfying strict quality criteria were aligned (Cf section below) and processed by RIPCAL [35]. RIPCAL output provides the number of transitions (Ti), transversions (Tv) and dinucleotide targets used in all possible transitions for each TE copy. These outputs were parsed to search for RIP signatures in TE copies and the dinucleotide targets used in the transition type mutations that are usually associated with a RIP-like mechanism. In addition, $R$ was used to generate graphics from REPET pipelines and RIPCAL results.

\section{Multiple sequence alignments of TE copies}

Multiple sequence alignments may be difficult to calculate if TE copies are fragmented and degenerated. To tackle this problem, we first performed pairwise alignments between each copy and its cognate consensus sequence, and then we used the consensus as a reference to derive a multiple alignment using refalign and refalign2fasta respectively (from REPET package). We filtered out the consensus families and TE copies $<400$ bases to produce a dataset satisfying the fixed quality criteria for RIPCAL. We also filtered out copies with less than $80 \%$ identity with the consensus sequence from the pairwise alignment and we excluded TE families with less than five sequences in the multiple alignments (TE consensus + four TE copies). In order to address the problem of degenerated and nested TE copies, the "long join procedure" implemented in the TEannot pipeline allow the connection of 2 ore more fragments to generate a TE copy.

\section{Analysis of GC content}

The GC content of TE copies and sliding windows along the genome (GSW) were calculated an in-house script that divided the genome into sliding windows and calculated the $\mathrm{GC}$ percentage $(\mathrm{GC} \%)$ in each window. $\mathrm{R}$ was used to calculate the distribution of TE copies and GSW according to the $\mathrm{GC} \%$.

\section{Search for proteins of the Dnmt1 family}

We used RID, Masc1, Masc2 and Dim-2 protein sequences [14] as queries for Blastp [51] analysis to search for genes encoding cytosine DNA methytransferase from the Dnmt1 subfamily in the fungal genomes studied. We first searched for genes with GeneName = $R I D$ or GeneName $=$ Masc 1 or GeneName $=$ Masc 2 in Uniprot using SRS at EBI (http://srs.ebi.ac.uk/). Of the 14 genes found, we kept six non-redundant ones (O13369_ASCIM Masc1 Ascobolus immersus; O42731_ ASCIM Masc2 A. immersus; B0B065_SORMA, RID, Sordaria macrospora; Q8NJV8_NEUTT, RID, Neurospora tetrasperma; Q8NJV9_NEUIN, RID, Neurospora intermedia; Q8NJW0_NEUCR, RID, Neurospora crassa). Using this set of six genes, we searched for similar genes in Uniprot with Blastp (e-value $<1 \mathrm{e}^{-10}$ ). We also searched the Microbotryum protein databank of the Broad Institute (http://www.broadinstitute.org/annotation/genome/Microb otryum_violaceum/MultiDownloads.html). We then filtered this new set of genes for redundancy particularly from re-sequenced species. We kept 30 non-redundant genes that were equally distributed along the tree. We then added the Dnmt1 genes found for the species in the tree (Additional file 1) to this set of genes, which gave a final list of 44 genes including all the RID/Masc1 and Masc2/Dim-2 genes of the 10 genomes of this study.

\section{Phylogenomic analysis of the 44 Dnmt 1 fungal proteins}

We performed an Interproscan analysis (Version 4.8 at http://www.ebi.ac.uk/Tools/pfa/iprscan/), [52] were to identify domains specific to each fungal Dnmt1 gene. The input parameters of this program were iprscan, Nocrc false, and GO terms true. The applications were blastprodom, fprintscan, hmmpir, hmmpfam, hmmsmart, hmmtigr, profilescan, hamap, patternscan, superfamily, signalp, tmhmm, hmmpanther, and gene3d (Additional file 5).

The PTHR10629 domain sequences from the Panther HMM domain database that were found in the studied genomes and other sequences from 15 ascomycetes and 8 basidiomycetes (including five and three of our study, respectively) were aligned with the $\mathrm{T}$-Coffee multiple sequence alignment program [53] and analyzed phylogenetically with PhyML [54]. The analysis was performed on the Phylogeny.fr platform [55] and comprised the following steps: (i) sequences were aligned with T-Coffee (v6.85) [53] with the following pair-wise alignment methods: the 10 best local alignments (Lalign_pair) and an accurate global alignment (slow_pair). (ii) After alignment, ambiguous regions (i.e. those containing gaps and/ or poorly aligned regions) were removed with Gblocks (v0.91b) [56] (using the following parameters: minimum length of a block after gap cleaning: 5 , positions with a gap in less than $50 \%$ of the sequences were selected in the final alignment if they were within an appropriate block, 
all segments with contiguous, not conserved positions bigger than 8 were rejected, minimum number of sequences for a flank position: 55\%. (iii) The phylogenetic tree was reconstructed using the maximum likelihood method implemented in the PhyML program (v3.0 aLRT) [57]. The WAG substitution model was selected assuming an estimated proportion of invariant sites (of 0.191) and four gamma-distributed rate categories to account for rate heterogeneity across sites. The gamma shape parameter was estimated directly from the data $($ gamma $=1.964)$. The reliability of the internal branch was assessed using the aLRT test (SH-Like). (iv) The phylogenetic tree was drawn and edited with TreeDyn software (v198.3) [58].

In addition we performed a MEME search to identify conserved motifs [59] (version 4.9.1) in the 44 proteins. The parameters used were -nmotifs 30, -minw 6 and -maxw 50. Of the 30 motifs found, we filtered out those present in at least one protein of the two subgroups of Dnmt1. Results (html files and motifs in logo format) are provided in Additional file 6.

\section{Additional files}

Additional file 1: Is a figure representing a phylogenetic tree of species used in DNMT1 phylogenetic analysis. Species used in RIP analysis are highlighted in blue. Genome name in Uniprot accession and abbreviation used in the manuscript (in blue) are in brackets. This tree was based on NCBI Taxonomy Browser (http://www.ncbi.nlm.nih.gov/ Taxonomy/taxonomyhome.html). Only the topology is shown and the branch lengths are not proportional to evolutionary divergence time.

Additional file 2: Is a figure representing a multiple sequence alignments (MSA) performed with T-Coffee (See Method section) for the C5_MTASE PFAM (PF000145) domain sequences extracted from the 44 Dnmt 1 proteins used in the phylogenetic analysis.

Additional file 3: Is a figure representing combined Block Diagrams found with MEME. Eleven out of the 30 motifs found were filtered out because they were present at least once in both the Dnmt 1 class I and class II subgroup (Figure 5A). Non-overlapping sites with a p-value below 0.0001 . The height of the motif "block" is proportional to the log(p-value); height is truncated for motifs with a p-value of 1e-10.

Additional file 4: Is a figure representing functional annotation of 44 fungal Dnmt1 proteins and phylogenetic analysis of the cytosine-specific methyltransferase domain. This figure results from superposition of Figure 5 and Additional file 3. (A) Phylogenetic analysis of 44 cytosine-specific methyltransferase domains (PTHR10629): Gray rectangles in 5B, including PF00145, PS00094, PS00095 et PR00105) from Dnmt1 fungal proteins and S. Pombe DNMT2, which was used as an outgroup. (B) Functional annotation of genes (white rectangle) with Interproscan (Cf Methods section). PTHR10629:SF10 is drawn on top of PTHR10629 (the coordinates are the same). Where PTHR10629 is not visible, it was overlapped by PF00145. Combined Block Diagrams found with MEME are superposed on functional domains found with Interproscan.

Additional file 5: Contains result of the interproscan analysis of proteins displayed in Figure 5.

Additional file 6: Contains html files corresponding to the results of the MEME analysis. index.html is the main page to open. Display comments when mouse pass over a domain.

\section{Competing interests}

The authors declare that they have no competing interests.

\section{Authors' contributions}

JA: designed the project and methods, carried out the analysis and wrote the manuscript. MHL: contributed to the design of the project and the writing of the manuscript. HQ: designed the project and methods and contributed to the writing of the manuscript. The following additional data are available with the online version of this paper. All authors read and approved the final manuscript.

\section{Acknowledgements}

We are grateful to the Broad Institute and particularly the leaders of the Microbotryum violaceum sequencing project (Michael Perlin, University of Louisville, David Schultz, University of Louisville, Christina Cuomo, Broad Institute) for generating and making available the genome assembly and the protein-coding gene data set, MVLG_04160, and for giving us permission to use this data in our study.

We would like to gratefully acknowledge Ludovic Mallet for the re-annotation of transposable elements in Magnaporthe oryzae.

We also are grateful to Florian Maumus for his helpful comments

subsequent to the critical reading of the manuscript.

Received: 9 February 2015 Accepted: 16 February 2015

Published online: 28 February 2015

\section{References}

1. Wicker T, Sabot F, Hua-Van A, Bennetzen JL, Capy P, Chalhoub B, et al. A unified classification system for eukaryotic transposable elements. Nat Rev Genet. 2007;8:973-82.

2. Coghlan A, Eichler EE, Oliver SG, Paterson AH, Stein L. Chromosome evolution in eukaryotes: a multi-kingdom perspective. Trends Genet. 2005:21:673-82.

3. Biemont C. A brief history of the status of transposable elements: from junk DNA to major players in evolution. Genetics. 2010;186:1085-93.

4. Manning VA, Pandelova I, Dhillon B, Wilhelm LJ, Goodwin SB, Berlin AM, et al. Comparative genomics of a plant-pathogenic fungus, Pyrenophora tritici-repentis, reveals transduplication and the impact of repeat elements on pathogenicity and population divergence. G3 (Bethesda). 2013;3:41-63.

5. Fudal I, Ross S, Brun H, Besnard AL, Ermel M, Kuhn ML, et al. Repeat-induced point mutation (RIP) as an alternative mechanism of evolution toward virulence in Leptosphaeria maculans. Mol Plant Microbe Interact. 2009;22:932-41.

6. Kim MY, Zilberman D. DNA methylation as a system of plant genomic immunity. Trends Plant Sci. 2014;19:320-6.

7. Jeffrey T, Selker EU. Gene silencing in filamentous fungi: RIP, MIP and quelling. J Genet. 1996;75:313-24.

8. Selker EU, Cambareri EB, Jensen BC, Haack KR. Rearrangement of duplicated DNA in specialized cells of Neurospora. Cell. 1987;51:741-52.

9. Cambareri EB, Jensen BC, Schabtach E, Selker EU. Repeat-induced G-C to A-T mutations in Neurospora. Science. 1989;244:1571-5.

10. Galagan JE, Selker EU. RIP: the evolutionary cost of genome defense. Trends Genet. 2004;20:417-23.

11. Neuveglise C, Sarfati J, Latge JP, Paris S. Afut1, a retrotransposon-like element from Aspergillus fumigatus. Nucleic Acids Res. 1996;24:1428-34.

12. Braumann I, van den Berg M, Kempken F. Repeat induced point mutation in two asexual fungi, Aspergillus niger and Penicillium chrysogenum. Curr Genet. 2008:53:287-97.

13. Clutterbuck AJ. Genomic evidence of repeat-induced point mutation (RIP) in filamentous ascomycetes. Fungal Genet Biol. 2011;48:306-26.

14. Jurkowski TP, Jeltsch A. On the evolutionary origin of eukaryotic DNA methyltransferases and Dnmt2. PLoS One. 2011;6:e28104.

15. Ponger L, Li WH. Evolutionary diversification of DNA Methyltransferases in eukaryotic Genomes. Mol Biol Evol. 2005;22:1119-28.

16. Freitag M, Williams RL, Kothe GO, Selker EU. A cytosine methyltransferase homologue is essential for repeat-induced point mutation in Neurospora crassa. Proc Natl Acad Sci U S A. 2002;99:8802-7.

17. Galagan JE, Calvo SE, Cuomo C, Ma LJ, Wortman JR, Batzoglou S, et al. Sequencing of Aspergillus nidulans and comparative analysis with $A$. fumigatus and A. oryzae. Nature. 2005:438:1105-15.

18. Cuomo CA, Gueldener U, Xu JR, Trail F, Turgeon BG, Di Pietro A, et al. The Fusarium graminearum genome reveals a link between localized polymorphism and pathogen specialization. Science. 2007;317:1400-2.

19. Rouxel T, Grandaubert J, Hane JK, Hoede C, van de Wouw AP, Couloux A, et al. Effector diversification within compartments of the Leptosphaeria 
maculans genome affected by Repeat-Induced Point mutations. Nat Commun. 2011;2:202.

20. Goyon C, Faugeron G. Targeted transformation of Ascobolus immersus and de novo methylation of the resulting duplicated DNA sequences. Mol Cell Biol. 1989;9:2818-27.

21. Malagnac F, Wendel B, Goyon C, Faugeron G, Zickler D, Rossignol JL, et al. A gene essential for de novo methylation and development in Ascobolus reveals a novel type of eukaryotic DNA methyltransferase structure. Cell. 1997;91:281-90.

22. Malagnac F, Gregoire A, Goyon C, Rossignol JL, Faugeron G. Masc2, a gene from Ascobolus encoding a protein with a DNA-methyltransferase activity in vitro, is dispensable for in vivo methylation. Mol Microbiol. 1999:31:331-8.

23. Romano N, Macino G. Quelling: transient inactivation of gene expression in Neurospora crassa by transformation with homologous sequences. Mol Microbiol. 1992;6:3343-53.

24. Fagard M, Boutet S, Morel JB, Bellini C, Vaucheret H. AGO1, QDE-2, and $\mathrm{RDE}-1$ are related proteins required for post-transcriptional gene silencing in plants, quelling in fungi, and RNA interference in animals. Proc Natl Acad Sci U S A. 2000:97:11650-4.

25. Fulci V, Macino G. Quelling: post-transcriptional gene silencing guided by small RNAs in Neurospora crassa. Curr Opin Microbiol. 2007:10:199-203.

26. Shiu PK, Raju NB, Zickler D, Metzenberg RL. Meiotic silencing by unpaired DNA. Cell. 2001;107:905-16.

27. Kouzminova E. Selker EU: dim-2 encodes a DNA methyltransferase responsible for all known cytosine methylation in Neurospora. Embo J. 2001;20:4309-23.

28. Chernov AV, Vollmayr P, Walter J, Trautner TA. Masc2, a C5-DNAmethyltransferase from Ascobolus immersus with similarity to methyltransferases of higher organisms. Biol Chem. 1997;378:1467-73.

29. Johnson LJ, Giraud T, Anderson R, Hood ME. The impact of genome defense on mobile elements in Microbotryum. Genetica. 2010;138:313-9.

30. Cooper DN, Youssoufian H. The CpG dinucleotide and human genetic disease. Hum Genet. 1988:78:151-5.

31. Horns F, Petit E, Yockteng R, Hood ME. Patterns of repeat-induced point mutation in transposable elements of basidiomycete fungi. Genome Biol Evol. 2012:4:240-7.

32. Flutre T, Duprat E, Feuillet C, Quesneville H. Considering transposable element diversification in de novo annotation approaches. PLoS One. 2011;6:e16526.

33. Hoede C, Arnoux S, Moisset M, Chaumier T, Inizan O, Jamilloux V, et al, PASTEC: an automatic transposable element classification tool. PLoS One. 2014:9:e91929.

34. Quesneville H, Bergman CM, Andrieu O, Autard D, Nouaud D, Ashburner M, et al. Combined evidence annotation of transposable elements in genome sequences. PLoS Comput Biol. 2005;1:166-75.

35. Hane JK, Oliver RP. RIPCAL: a tool for alignment-based analysis of repeat-induced point mutations in fungal genomic sequences. BMC Bioinformatics. 2008;9:478.

36. Amselem J, Cuomo CA, van Kan JA, Viaud M, Benito EP, Couloux A, et al. Genomic analysis of the necrotrophic fungal pathogens Sclerotinia sclerotiorum and Botrytis cinerea. PLoS Genet. 2011;7:e1002230.

37. Spanu PD, Abbott JC, Amselem J, Burgis TA, Soanes DM, Stuber K, et al. Genome expansion and gene loss in powdery mildew fungi reveal tradeoffs in extreme parasitism. Science. 2010;330:1543-6.

38. Dean RA, Talbot NJ, Ebbole DJ, Farman ML, Mitchell TK, Orbach MJ, et al. The genome sequence of the rice blast fungus Magnaporthe grisea. Nature. 2005:434:980-6.

39. Martin F, Kohler A, Murat C, Balestrini R, Coutinho PM, Jaillon O, et al. Perigord black truffle genome uncovers evolutionary origins and mechanisms of symbiosis. Nature. 2010;464:1033-8.

40. Duplessis S, Cuomo CA, Lin YC, Aerts A, Tisserant E, Veneault-Fourrey C, et al. Obligate biotrophy features unraveled by the genomic analysis of rust fungi. Proc Natl Acad Sci U S A. 2011;108:9166-71.

41. Raffaele S, Kamoun S. Genome evolution in filamentous plant pathogens: why bigger can be better. Nat Rev Microbiol. 2012;10:417-30

42. Wicker T, Oberhaensli S, Parlange F, Buchmann JP, Shatalina M, Roffler S, et al. The wheat powdery mildew genome shows the unique evolution of an obligate biotroph. Nat Genet. 2013;45(9):1092-6. advance online publication.
43. Ahmed I, Sarazin A, Bowler C, Colot V, Quesneville H. Genome-wide evidence for local DNA methylation spreading from small RNA-targeted sequences in Arabidopsis. Nucleic Acids Res. 2011;39:6919-31.

44. Maumus F, Quesneville $\mathrm{H}$. Deep investigation of Arabidopsis thaliana junk DNA reveals a continuum between repetitive elements and genomic dark matter. PLoS One. 2014:9:e94101.

45. Buisine N, Quesneville H, Colot V. Improved detection and annotation of transposable elements in sequenced genomes using multiple reference sequence sets. Genomics. 2008;91:467-75.

46. Callebaut I, Courvalin JC, Mornon JP. The BAH (bromo-adjacent homology) domain: a link between DNA methylation, replication and transcriptional regulation. FEBS Lett. 1999:446:189-93.

47. Rountree MR, Bachman KE, Baylin SB. DNMT1 binds HDAC2 and a new co-repressor, DMAP1, to form a complex at replication foci. Nat Genet. 2000;25:269-77.

48. O'Connell RJ, Thon MR, Hacquard S, Amyotte SG, Kleemann J, Torres MF, et al. Lifestyle transitions in plant pathogenic Colletotrichum fung deciphered by genome and transcriptome analyses. Nat Genet. 2012:44:1060-5.

49. Montanini B, Chen PY, Morselli M, Jaroszewicz A, Lopez D, Martin F, et al. Non-exhaustive DNA methylation-mediated transposon silencing in the black truffle genome, a complex fungal genome with massive repeat element content. Genome Biol. 2014;15:411.

50. Jurka J, Kapitonov W, Pavlicek A, Klonowski P, Kohany O, Walichiewicz J. Repbase Update, a database of eukaryotic repetitive elements. Cytogenet Genome Res. 2005;110:462-7.

51. Altschul SF, Gish W, Miller W, Myers EW, Lipman DJ. Basic local alignment search tool. J Mol Biol. 1990:215:403-10.

52. Quevillon E, Silventoinen V, Pillai S, Harte N, Mulder N, Apweiler R, et al. InterProScan: protein domains identifier. Nucleic Acids Res. 2005;33:W116-20.

53. Notredame C, Higgins DG, Heringa J. T-Coffee: a novel method for fast and accurate multiple sequence alignment. J Mol Biol. 2000;302:205-17.

54. Anisimova M, Gascuel O. Approximate likelihood-ratio test for branches: a fast, accurate, and powerful alternative. Syst Biol. 2006;55:539-52.

55. Dereeper A, Guignon V, Blanc G, Audic S, Buffet S, Chevenet F, et al. Phylogeny.fr: robust phylogenetic analysis for the non-specialist. Nucleic Acids Res. 2008;36:W465-9.

56. Castresana J. Selection of conserved blocks from multiple alignments for their use in phylogenetic analysis. Mol Biol Evol. 2000;17:540-52.

57. Guindon S, Gascuel O. A simple, fast, and accurate algorithm to estimate large phylogenies by maximum likelihood. Syst Biol. 2003;52:696-704.

58. Chevenet F, Brun C, Banuls AL, Jacq B, Christen R. TreeDyn: towards dynamic graphics and annotations for analyses of trees. BMC Bioinformatics. 2006;7:439.

59. Bailey $T L$, Elkan C. Fitting a mixture model by expectation maximization to discover motifs in biopolymers. Proc Int Conf Intell Syst Mol Biol. 1994;2:28-36.

\section{Submit your next manuscript to BioMed Central and take full advantage of:}

- Convenient online submission

- Thorough peer review

- No space constraints or color figure charges

- Immediate publication on acceptance

- Inclusion in PubMed, CAS, Scopus and Google Scholar

- Research which is freely available for redistribution 